\title{
Immune Checkpoint Inhibitors
} Regulate $\mathrm{K}^{+}$Channel Activity in Cytotoxic T Lymphocytes of Head and Neck Cancer Patients

\section{OPEN ACCESS}

Edited by:

Ildikò Szabò,

University of Padua, Italy

Reviewed by:

Timm Danker,

University of Tübingen, Germany

Hongguang Nie,

China Medical University, China

*Correspondence:

Laura Conforti

laura.conforti@uc.edu

Specialty section:

This article was submitted to

Pharmacology of lon Channels and

Channelopathies,

a section of the journal

Frontiers in Pharmacology

Received: 16 July 2021 Accepted: 16 August 2021

Published: 27 August 2021

Citation:

Gawali VS, Chimote AA, Newton HS, Feria-Garzón MG, Chirra M, Janssen EM, Wise-Draper TM and Conforti L (2021) Immune Checkpoint Inhibitors Regulate $\mathrm{K}^{+}$Channel Activity in Cytotoxic T Lymphocytes of Head and Neck Cancer Patients.

Front. Pharmacol. 12:742862.

doi: 10.3389/fphar.2021.742862

\begin{abstract}
Vaibhavkumar S. Gawali ${ }^{1}$, Ameet A. Chimote ${ }^{1}$, Hannah S. Newton ${ }^{1}$, Manuel G. Feria-Garzón ${ }^{1}$, Martina Chirra ${ }^{1}$, Edith M. Janssen ${ }^{2,3}$, Trisha M. Wise-Draper ${ }^{4}$ and Laura Conforti ${ }^{1 *}$

${ }^{1}$ Department of Internal Medicine, Division of Nephrology, University of Cincinnati, Cincinnati, $\mathrm{OH}$, United States, ${ }^{2}$ Division of Immunobiology, Cincinnati Children's Hospital Medical Center, Cincinnati, OH, United States, ${ }^{3} / m m u n o l o g y$, Janssen Research and Development, Spring House, PA, United States, ${ }^{4}$ Department of Internal Medicine, Division of Hematology Oncology,

University of Cincinnati, Cincinnati, $\mathrm{OH}$, United States
\end{abstract}

Programmed death receptor-1 (PD-1) and its ligand (PD-L1) interaction negatively regulates $T$ cell function in head and neck squamous cell carcinoma (HNSCC). Overexpression of PD-1 reduces intracellular $\mathrm{Ca}^{2+}$ fluxes, and thereby $\mathrm{T}$ cell effector functions. In HNSCC patients, PD-1 blockade increases KCa3.1 and Kv1.3 activity along with $\mathrm{Ca}^{2+}$ signaling and mobility in $\mathrm{CD}^{+}$peripheral blood T cells (PBTs). The mechanism by which PD-L1/PD-1 interaction regulates ion channel function is not known. We investigated the effects of blocking PD-1 and PD-L1 on ion channel functions and intracellular $\mathrm{Ca}^{2+}$ signaling in $\mathrm{CD}^{+}$PBTs of HNSCC patients and healthy donors (HDs) using single-cell electrophysiology and live microscopy. Anti-PD-1 and anti-PD-L1 antibodies increase KCa3.1 and Kv1.3 function in $\mathrm{CD}^{+}$PBTs of HNSCC patients. Anti-PD-1 treatment increases $\mathrm{Ca}^{2+}$ fluxes in a subset of HSNCC patients. In CD8 ${ }^{+}$ PBTs of HDs, exposure to PD-L1 reduces $\mathrm{KCa} 3.1$ activity and $\mathrm{Ca}^{2+}$ signaling, which were restored by anti-PD-1 treatment. The PD-L1-induced inhibition of KCa3.1 channels was rescued by the intracellular application of the PI3 kinase modulator phosphatidylinositol 3phosphate (PI3P) in patch-clamp experiments. In HNSCC CD8 ${ }^{+}$PBTs, anti-PD-1 treatment did not affect the expression of $\mathrm{KCa} 3.1, \mathrm{Kv1} .3, \mathrm{Ca}^{2+}$ release activated $\mathrm{Ca}^{2+}$ (CRAC) channels, and markers of cell activation (CD69) and exhaustion (LAG-3 and TIM3). Our data show that immune checkpoint blockade improves $T$ cell function by increasing KCa3.1 and Kv1.3 channel activity in HNSCC patients.

Keywords: ion channels, immune checkpoint inhibitors, $\mathrm{KCa} .1$ (intermediate-conductance $\mathrm{Ca}^{2+}$-activated $\mathrm{K}^{+}$ channel), Kv1.3 channel, $\mathrm{Ca}^{2+}$ signalling, head and neck (H\&N) cancer, immunotharapy

\section{INTRODUCTION}

Head and neck squamous cell cancer (HNSCC) is the seventh most common cancer worldwide (Chow, 2020; Sung et al., 2021). Conventional treatments include surgery, radiotherapy, chemotherapy, and multimodal approaches. However, the prognosis of locally advanced disease remains poor, with a 5 years overall survival $<50 \%$ (Chow, 2020). The discovery of immunotherapy has changed the landscape of HNSCC treatment by offering long term 
response in a subset of patients. Programmed cell death receptor-1 (PD-1) immune checkpoint inhibitors have shown promising responses in the treatment of HNSCC. Hence, they have been approved by the FDA since 2016 (Sharma et al., 2017; Chow, 2020). However, over $80 \%$ of HNSCC patients do not respond to PD-1 blockade (Bauml et al., 2019). Resistance to immunotherapy remains a big challenge as the majority of patients do not show a response to the treatment (primary resistance) (Sharma et al., 2017). Furthermore, some patients who initially respond and receive benefit from the treatment, later show disease relapse (acquired resistance) (Sharma et al., 2017). Therefore, better understanding of PD-1 signaling is necessary to develop new therapeutic options. The interaction of PD-1 ligand -1 and 2 (PD-L1/2) with their receptor $\mathrm{PD}-1$ on cytotoxic $\mathrm{T}$ cells negatively regulates $\mathrm{T}$ cell function and causes apoptosis, anergy, and exhaustion (Zhou et al., 2017). PD-L1 is constitutively present in T cells and other immune cells, and it's over expression by tumor cells contributes to immune evasion (Zhou et al., 2017). PD-L1 is also present, and biologically active, in the plasma of HNSCC patients in a secreted form and bound to exosomes contributing to immune evasion (Theodoraki et al., 2018). However, the mechanisms of PD-L1-mediated PD-1 signaling on $\mathrm{T}$ cell function are not fully understood.

Ion channels in $\mathrm{T}$ lymphocytes orchestrate the influx of intracellular $\mathrm{Ca}^{2+}$ required for downstream effector functions, such as migration and proliferation. Calcium release activated $\mathrm{Ca}^{2+}$ (CRAC), voltage-gated $\mathrm{K}^{+}(\mathrm{Kv} 1.3)$ and $\mathrm{Ca}^{2+}$ activated $\mathrm{K}^{+}$ (KCa3.1) channels are involved in the early phase of $\mathrm{T}$ cell activation and regulate the $\mathrm{Ca}^{2+}$ influx necessary for their effector functions. $\mathrm{Ca}^{2+}$ influx is triggered by the $\mathrm{T}$ cell receptor (TCR) mediated-depletion of $\mathrm{Ca}^{2+}$ stores in the endoplasmic reticulum and opening of the CRAC channels, and it is aided by Kv1.3 and KCa3.1 channels, which allow maintenance of the negative membrane potential necessary for $\mathrm{Ca}^{2+}$ influx through the CRAC channels (Feske et al., 2015). KCa3.1 channels also control chemotaxis (Chimote et al., 2018; Chimote et al., 2020). Defective ion channel function leads to alterations in $\mathrm{Ca}^{2+}$ signaling and downstream effector functions (Feske et al., 2006). In HNSCC patients, reduced expression of Kv1.3 in tumor infiltrating lymphocytes (TILs) contributes to lower $\mathrm{Ca}^{2+}$ response and cytotoxicity (Chimote et al., 2017). Furthermore, reduced activity of $\mathrm{KCa} 3.1$ in $\mathrm{CD}^{+}$peripheral blood T cells (PBTs) of HNSCC patients causes these cells to be hypersensitive to adenosine found in the tumor microenvironment, ultimately reducing their migratory abilities and restricting their infiltration into tumors (Chimote et al., 2013; Chimote et al., 2018).

It has been reported that overexpression of $\mathrm{PD}-1$ influences TCR-dependent effector functions, such as $\mathrm{Ca}^{2+}$ fluxing, secretion of cytokines and cytotoxic activity (Wei et al., 2013). Furthermore, TILs of melanoma patients with high expression of PD-1 have decreased $\mathrm{Ca}^{2+}$ responses to TCR stimulation (Chapon et al., 2011). We have recently shown that treatment of HNSCC patients with pembrolizumab, a monoclonal blocking antibody against PD1 (aPD-1), increases KCa3.1 and Kv1.3 activities in $\mathrm{CD}^{+}$TILs and PBTs, along with their $\mathrm{Ca}^{2+}$
TABLE 1 | Demographic and clinical data of HNSCC patients enrolled in the study.

\begin{tabular}{|c|c|}
\hline Age (at the time of sample collection) & Years \\
\hline Range & $18-90$ \\
\hline Mean & 59 \\
\hline Variable & Number (\%) \\
\hline \multicolumn{2}{|l|}{ Sex } \\
\hline Male & $23(71)$ \\
\hline Female & $9(39)$ \\
\hline \multicolumn{2}{|l|}{ Site } \\
\hline Oral Cavity & $10(31)$ \\
\hline Oropharynx & $14(43)$ \\
\hline Larynx & $07(21)$ \\
\hline Hypopharynx & $0(0)$ \\
\hline Nasopharynx & $0(0)$ \\
\hline Unknown Primary & $1(3)$ \\
\hline \multicolumn{2}{|l|}{ Primary Tumor } \\
\hline T1 & $8(25)$ \\
\hline T2 & $10(31)$ \\
\hline T3 & $5(16)$ \\
\hline $\mathrm{T} 4$ & $8(25)$ \\
\hline Unknown & $1(3)$ \\
\hline \multicolumn{2}{|l|}{ Nodal Status } \\
\hline NO & $7(21)$ \\
\hline $\mathrm{N} 1$ & $11(34)$ \\
\hline N2 & $11(34)$ \\
\hline N3 & $3(9)$ \\
\hline Unknown & $0(0)$ \\
\hline \multicolumn{2}{|l|}{ ECOG performance status } \\
\hline 0 & $6(19)$ \\
\hline 1 & $13(41)$ \\
\hline 2 & $4(12)$ \\
\hline 3 & $1(3)$ \\
\hline Unknown & $8(25)$ \\
\hline \multicolumn{2}{|l|}{ Smoking } \\
\hline No (<10 pack years) & $14(44)$ \\
\hline Yes (>10 pack years) & $18(56)$ \\
\hline \multicolumn{2}{|l|}{ Alcohol } \\
\hline No (<5 drinks/week) & $22(69)$ \\
\hline Yes (>5 drinks/week) & $9(28)$ \\
\hline Unknown & $1(3)$ \\
\hline \multicolumn{2}{|l|}{ p16 status } \\
\hline Positive & $12(38)$ \\
\hline Negative & $13(41)$ \\
\hline Unknown & $7(22)$ \\
\hline
\end{tabular}

HNSCC patients $(\mathrm{n}=32)$ were enrolled in the study upon fulfillment of eligibility criteria. TNM staging system was used to stage tumor size and nodal involvement. T1 to T4 refers to the size and invasion of the tumors. N1 to N3 refers to the assessment of number and location of the regional lymph nodes. The ECOG (Eastern Cooperative Oncology Group) performance status indicates daily quality of life of individuals affected by diseases on a scale of 0-5. Smoking status (pack years) was calculated by multiplying the number of packs of cigarettes smoked per day by the number of years the person has smoked. We used a cutoff of 10 packs per year to differentiate the smoking status.

fluxing and migratory abilities (Newton et al., 2020). Furthermore, a specific pattern of $\mathrm{K}^{+}$channel reinvigoration was associated with a pathological response to therapy (Newton et al., 2020). However, these studies did not allow us to dissect the mechanisms by which PD-1 signaling affects ion channel activity. Herein, we conducted in vitro studies that further our understanding of the interconnection between PD1 and ion channels in T cells. These studies showed that blockade of PD-L1/PD-1 interaction leads to the rapid activation of KCa3.1 and Kv1.3 channels to ultimately regulate $\mathrm{Ca}^{2+}$ signaling in HNSCC patients' $T$ cells. 


\section{MATERIALS AND METHODS}

\section{Human Subjects}

Peripheral blood samples from de-identified HNSCC patients $(n=$ 32) were obtained from the University of Cincinnati Medical Center. HNSCC patients included in this study were treatmentnaive and had a positive diagnosis of HNSCC by tissue biopsy (See Table 1 for a summary of patient demographics and Supplementary Table S1 for clinical information). Peripheral blood samples of 7 healthy donors (HDs, 4 males and 3 females, age range between 30 and 65 years) were collected from individual donors and from discarded blood units (Hoxworth Blood Center, University of Cincinnati). The demographics of the donors from Hoxworth Blood center were not available. Informed consent was obtained from all HNSCC patients and HDs. The data collected in the study were managed using the Research Electronic Data Capture (REDCap) tools licensed to the University of Cincinnati. Sample collection was approved by the University of Cincinnati Institutional Review Board (IRB no. 2014-4755).

\section{Reagents and Chemicals}

Human serum, L-glutamine, sodium hydroxide, poly-L-lysine, LY294002 HCl, ionomycin, calmodulin, poly-L-lysine, thapsigargin (TG), tetraethylammonium-chloride (TEA-Cl), 1,2-Bis(2-Aminophenoxy)ethane- $N, N, N^{\prime}, N^{\prime}$-tetraacetic acid (BAPTA), $\mathrm{MgCl}_{2}$ were purchased from Millipore Sigma. Sterile, 4-(2-hydroxyethyl)-1-piperazineethanesulfonic acid (HEPES), RPMI-1640 medium, fetal bovine serum, penicillin, streptomycin, Fura-2 and phosphate buffered saline (PBS) were obtained from ThermoFisher. Phosphatidylinositol 3-phosphate diC16 (PI3P) was purchased from Echelon Biosciences. Pembrolizumab (Merck Sharpe and Dohme Corp) samples used in this study were received from the leftover pharmacy supply at Cincinnati Children's Hospital. Atezolizumab was purchased from Biovision life sciences. PD-L1-Fc chimera was obtained from R\&D systems. Stock solutions of LY394002 and TG were prepared in dimethyl sulfoxide and used at $0.1 \%$ dilution. Stock solutions of PD-L1-Fc, pembrolizumab and atezolizumab were prepared in sterile PBS.

\section{Cell isolation and in vitro Activation}

Peripheral blood mononuclear cells were isolated from whole blood by Ficoll-Paque density gradient centrifugation (Cytiva) as previously described (Chimote et al., 2018). CD8 ${ }^{+}$PBTs were isolated by negative selection using EasySep Human $\mathrm{CD}^{+} \mathrm{T}$ cell Enrichment kit (StemCell Technologies). Post isolation, $\mathrm{CD}^{+}$ PBTs were maintained in RPMI-1640 medium supplemented with $10 \%$ human serum, $200 \mathrm{U} / \mathrm{ml}$ penicillin, $200 \mathrm{mg} / \mathrm{ml}$ streptomycin, $1 \mathrm{mM}$-glutamine, and $10 \mathrm{mM}$ HEPES. Activation of cells was performed either by adding $40.5 \mathrm{nM}$ of phorbol-12-myristate-13-acetate (PMA, Millipore Sigma) and $1.5 \mu \mathrm{M}$ of ionomycin (Millipore Sigma) or in cell culture plates coated with $10 \mu \mathrm{g} / \mathrm{ml}$ anti-CD3 and anti-CD28 antibodies (BioLegend) for $72-96 \mathrm{~h}(\mathrm{~h})$ as previously described (Chimote et al., 2016; Chimote et al., 2018). After isolation, some fresh cells were used for functional studies, the remaining cells were frozen and used later on for flow cytometry experiments.

\section{Treatment With aPD-1/PD-L1 Antibodies and PD-L1-FC}

$\mathrm{CD}^{+}$PBTs from HNSCC patients were activated for $72-96 \mathrm{~h}$ using PMA and ionomycin followed by treatment with the aPD-1 antibody pembrolizumab $(10 \mu \mathrm{g} / \mathrm{ml})$ and/or the $a \mathrm{PD}-\mathrm{L} 1$ antibody atezolizumab ( 1 and $10 \mu \mathrm{g} / \mathrm{ml}$ ) for $6 \mathrm{~h}$ prior to performing functional studies. $\mathrm{CD}^{+} \mathrm{PBTs}$ from $\mathrm{HDs}$ were plated on cell culture plates coated with PD-L1 (PD-L1-Fc, R\&D Systems) $(10 \mu \mathrm{g} / \mathrm{ml})$ and activated with either PMA and ionomycin or (with anti-CD3/CD28 antibodies for $72-96 \mathrm{~h}$ followed by treatment with the $\alpha \mathrm{PD}-1$ antibody pembrolizumab $(10 \mu \mathrm{g} / \mathrm{ml})$ for $6 \mathrm{~h}$ before performing functional studies. For prolonged treatment with PD-L1, cells were activated for $120 \mathrm{~h}$ with plate-bound anti-CD3/CD8 antibodies along with PD-L1-Fc in $\mathrm{T}$ cell medium supplemented with $20 \mathrm{IU} / \mathrm{ml}$ of IL-2.

\section{Electrophysiology}

Whole cell patch-clamp electrophysiology was used to measure the activity of KCa3.1 and Kv1.3 channels in activated CD8 ${ }^{+}$ PBTs cells of HDs and HNSCC patients as described previously (Chimote et al., 2020). The external solution consisted of (in $\mathrm{mM}$ ): $160 \mathrm{NaCl}, 4.5 \mathrm{KCl}, 1 \mathrm{MgCl}_{2}, 10 \mathrm{HEPES}, \mathrm{pH}$ 7.4. Internal solution consisted of (in $\mathrm{mM}$ ): $145 \mathrm{~K}$-Aspartate, $2 \mathrm{MgCl}_{2}, 8.5$ $\mathrm{CaCl}_{2}, 10$ EGTA, 10 HEPES, pH 7.2 TRIS buffer $\left(1 \mu \mathrm{M}\right.$ free $\mathrm{Ca}^{2+}$ concentration). Borosilicate glass (World Precision Instruments) pipettes (4-5 M $\Omega$ resistance) were fabricated using a P-97 horizontal puller (Sutter Instruments). A voltage-ramp pulse protocol (from -120 to $+50 \mathrm{mV}$, for $200 \mathrm{~ms}$, holding potential of $-70 \mathrm{mV}$, every $15 \mathrm{~s}$ ) was used to elicit the currents from CD8 ${ }^{+}$ PBTs. Currents were recorded, amplified and digitalized (Axon 200B and Digidata 1320A, Molecular Devices) through a 16-bit A-D/D-A interface. Data acquisition was performed using pClamp 8.0 software (Molecular Devices) and signals were low pass filtered at $2 \mathrm{kHz}$ and digitalized at $100 \mathrm{kHz}$. The conductance (G) of KCa3.1 channels was calculated as, the ratio of linear fraction of the currents to the slope of ramp voltage stimulus (measured in the voltage range between -100 and $-80 \mathrm{mV}$ ) after subtraction of leak current. (Chimote et al., 2018). The G of Kv1.3 channels was determined from the same recordings by measuring the peak currents at $+50 \mathrm{mV}$ after subtraction of KCa3.1 currents extrapolated by linear regression. This protocol accurately record and separate KCa3.1 and Kv1.3 currents (Newton et al., 2020). LY294002 was delivered extracellular via a manual perfusion system. Calmodulin and PI3P were dissolved in the internal solution and delivered intracellularly via the patch-pipette. The KCa3.1 and Kv1.3 Gs were measured in at least three to five cells for each condition per individual patient.

For divalent free (DVF) currents, cells patched in the wholecell configuration were pre-incubated with TG $(1 \mu \mathrm{M})$ in external solution without $\mathrm{Ca}^{2+}$ for $10 \mathrm{~min}$ followed by perfusion with $20 \mathrm{mM} \mathrm{Ca}^{2+}$ for $1 \mathrm{~min}$ and, finally, with DVF solution for $2 \mathrm{~min}$ (Vaeth et al., 2017). DVF currents were measured to amplify the CRAC currents. The DVF Ringer's solution contained (in $\mathrm{mM}$ ): $150 \mathrm{NaCl}, 10$ HEDTA, 1 EDTA and 10 HEPES (pH 7.4 with $\mathrm{NaOH}$ ). The $20 \mathrm{mM} \mathrm{Ca}^{2+}$ external solution consisted of (in $\mathrm{mM}$ ): 
$130 \mathrm{NaCl}, 4.5 \mathrm{KCl}, 20 \mathrm{CaCl}_{2}, 10 \mathrm{D}$-glucose and 5 HEPES (pH 7.4 with $\mathrm{NaOH}$ ). $10 \mathrm{mM}$ TEA-Cl was added to all extracellular solutions to block voltage-gated $\mathrm{K}^{+}$channels. The pipette solution contained (in $\mathrm{mM}$ ): $135 \mathrm{Cs}$ aspartate, $8 \mathrm{MgCl}_{2}, 8$ BAPTA and 1 HEPES (pH 7.2 with $\mathrm{CsOH}$ ). A ramp (-100 to $+100 \mathrm{mV}$, holding potential of $+30 \mathrm{mV}$ ) protocol was used for $100 \mathrm{~m}$ every $1.5 \mathrm{~s}$ to record DVF currents. Analysis of DVF current was performed by measuring the peak current value at the voltage of $-100 \mathrm{mV}$ during the ramp step protocol.

\section{$\mathrm{Ca}^{2+}$ Flux Measurements}

Intracellular $\mathrm{Ca}^{2+}$ fluxes were measured in activated $\mathrm{CD} 8^{+} \mathrm{PBTs}$ using the ratiometric $\mathrm{Ca}^{2+}$ sensitive dye Fura-2. Perfusion with the sarco-endoplasmic pump inhibitor TG allowed us to measure $\mathrm{Ca}^{2+}$ fluxes that are independent of TCR stimulation and only rely on downstream signaling events initiated by the release of $\mathrm{Ca}^{2+}$ from the endoplasmic reticulum (Robbins et al., 2005). $\mathrm{T}$ cells were plated on poly-L-lysine coated coverslips followed by treatment with $1 \mu \mathrm{M}$ Fura-2 $\mathrm{AM}$ (ThermoFisher) at $37^{\circ} \mathrm{C}$ for $\sim 30 \mathrm{~min}$. Cells were then washed with RPMI-1640 and maintained at $37^{\circ} \mathrm{C}$ prior to recordings using $\operatorname{InCyt}-\operatorname{Im} 2 \mathrm{Ca}^{2+}$ imaging system (Intracellular Imaging). Coverslips were mounted on the microscope and perfused with a $\mathrm{Ca}^{2+}$ free solution for $5 \mathrm{~min}$, followed by perfusion with $1 \mu \mathrm{MTG}$ in $\mathrm{Ca}^{2+}$ free solution for other 5 min to deplete the $\mathrm{Ca}^{2+}$ from the intracellular $\mathrm{Ca}^{2+}$ stores and open the CRAC channels. Finally, $0.5 \mathrm{mM} \mathrm{Ca}^{2+}$ solution was perfused for $10-15 \mathrm{~min}$ to allow $\mathrm{Ca}^{2+}$ influx through CRAC channels. $\mathrm{Ca}^{2+}$ free solution had the following composition (in $\mathrm{mM}$ ): $155 \mathrm{NaCl}, 4.5 \mathrm{KCl}, 1 \mathrm{MgCl}_{2}$, 10 HEPES, 10 glucose, 2 EGTA, pH 7.4. The $0.5 \mathrm{mM} \mathrm{Ca}^{2+}$ solution had the following composition (in $\mathrm{mM}$ ): $155 \mathrm{NaCl}$, $4.5 \mathrm{KCl}, 2.5 \mathrm{MgCl}_{2}, 10$ HEPES, 10 glucose, $0.5 \mathrm{CaCl}_{2}$, pH 7.4. A standard calibration curve was used to correlate ratiometric Fura-2 values $\left(340 / 380 \mathrm{~nm}\right.$ ratio) with known $\mathrm{Ca}^{2+}$ concentrations as per the protocol provided by the manufacturer. Changes in $\mathrm{Ca}^{2+}$ values [Delta $(\Delta) \mathrm{Ca}^{2+}$, a measure of $\mathrm{Ca}^{2+}$ fluxing ability, were determined as the difference between the peak of $\mathrm{Ca}^{2+}$ reached after $0.5 \mathrm{mM}$ $\mathrm{Ca}^{2+}$ and the baseline $\mathrm{Ca}^{2+}$ after the perfusion with $1 \mu \mathrm{MTG}$ in $\mathrm{Ca}^{2+}$ free solution, immediately before the addition of $0.5 \mathrm{mM}$ $\mathrm{Ca}^{2+}$. Statistical analysis was performed to detect significant difference in $\Delta \mathrm{Ca}^{2+}$ values post treatment for an individual patient or healthy donor. Only those donors who showed a statistically significant increase in the $\mathrm{Ca}^{2+}$ values before and after $a \mathrm{PD}-1$ were included in the positive response (PR) group while those who did not show any statistically significant increase were included in the no-response (NR) group. Individual single cell $\Delta \mathrm{Ca}^{2+}$ values from donors in the PR group and the NR group were then combined to detect significance differences between the two groups. This is because of low sample quantity and variable cell count per patient.

\section{Flow Cytometry}

$\mathrm{CD}^{+}$PBT cells were maintained at a density of $1 \times 10^{6}$ cells $/ \mathrm{mL}$ and stimulated with PMA/ionomycin for $72 \mathrm{~h}$. Cells were then rinsed with $1 x$ PBS, followed by staining for flow cytometry. The proportion of dead cells were determined using the Zombie UV fixable viability kit (Biolegend). The cells were then fixed with $1 \%$ paraformaldehyde (ThermoFisher), washed with 1x PBS and stained overnight with mouse anti-human anti-KCa3.1 biotinylated antibodies (clone 6 $\mathrm{Cl}$, Alomone). Then, the cells were washed with 1x PBS and incubated for $30 \mathrm{~min}$ at room temperature with the following anti-human antibodies (all from Biolegend): anti-CD8-PacificBlue (cloneHIT8A), anti-CD69APCFire750 (clone FN50), anti-LAG3-BV510 (clone 11C3C56), anti-PD1-BV605 (clone EH12. 2H7), anti-TIM3BV786 (clone F38-2F2), and anti-streptavidin-PECy7 (clone). Next, the cells were washed with $1 \mathrm{x}$ PBS, and permeabilized with $\mathrm{BD}$ Cytofix/Cytoperm kit (BD Biosciences) as per the manufacturer's instructions, and incubated for $30 \mathrm{~min}$ at $4^{\circ} \mathrm{C}$ with mouse anti-human anti-Calmodulin-PerCP (clone 2D1, NOVUS Biologicals), and anti-human anti-Ki67-BV711 (Biolegend).

For determination of ion channel expression, cells were fixed with 4\% paraformaldehyde, washed with PBS followed by overnight incubation at $4^{\circ} \mathrm{C}$ with the following primary antibodies, mouse anti-human KCa3.1 (6C1/ATTO-488), guinea pig anti-human Kv1.3, rabbit anti-human Orail (all from Alomone labs) followed by anti-guinea pig (Dy350 goat anti-guinea pig IgG/Thermo Fisher) and anti-rabbit (Alexa Fluor 594 goat anti-rabbit IgG/Thermo Fisher) secondary antibodies. To stain for intracellular ion channel epitopes, cells were permeabilized with BD Cytofix/Cytoperm kit (BD Biosciences) as per manufacturer's instructions. Cells were then stained for rabbit anti-human STIM1 (Proteintech) primary antibodies followed by secondary antibodies (Alexa Fluor 594 goat antirabbit IgG/Thermo Fisher). Specificity of these antibodies was previously reported by our laboratory (Chimote et al., 2018; Newton et al., 2020). All flow cytometry data were collected on LSR Fortessa or LSR II flow cytometer (BD Biosciences), using the FACS Diva software v.6.0. At least 30,000 total events were acquired. Fluorescence minus one controls were also included. The flow cytometry data were analyzed using FlowJo Software version 10.6.1 ((BD Biosciences).

\section{Statistical Analysis}

Statistical analyses were performed using Student's $t$ test (paired or unpaired), Mann-Whitney rank sum test (in experiments where samples failed normality or had unequal variance), and ANOVA or ANOVA on Ranks as indicated. Post hoc testing on ANOVA was done by multiple pairwise comparison procedures using the Holm-Sidak, Dunn's or Tukey's methods depending on sample normality and variance. Statistical analysis was performed using SigmaPlot 13.0 (Systat Software Inc.). $p$ value of less than or equal to 0.05 was considered as statistically significant.

\section{RESULTS}

\section{PD-1 Blockade improves KCa3.1 and Kv1.3 Activity in $\mathrm{CD}^{+}$PBTs of HNSCC Patients}

Ion channels are fundamental regulators of $\mathrm{T}$ cell $\mathrm{Ca}^{2+}$ signaling and effector functions (Feske et al., 2015) and we have shown that treatment of HNSCC patients with pembrolizumab increases 

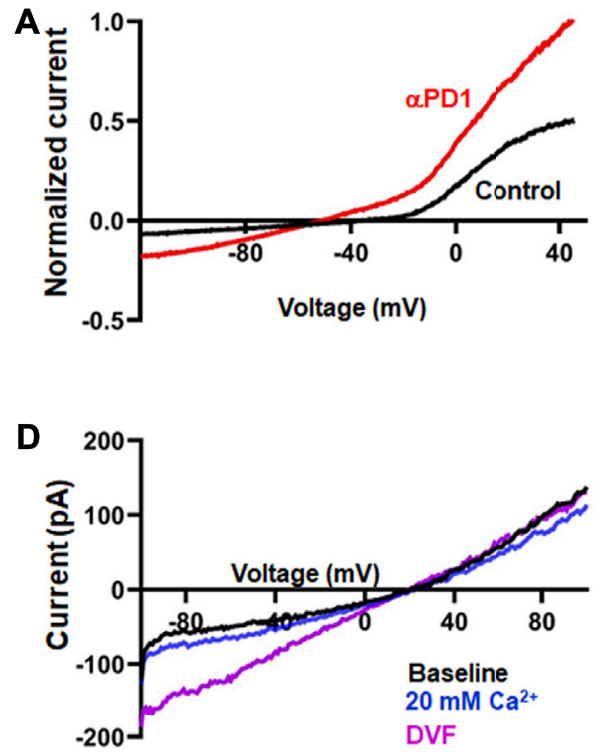
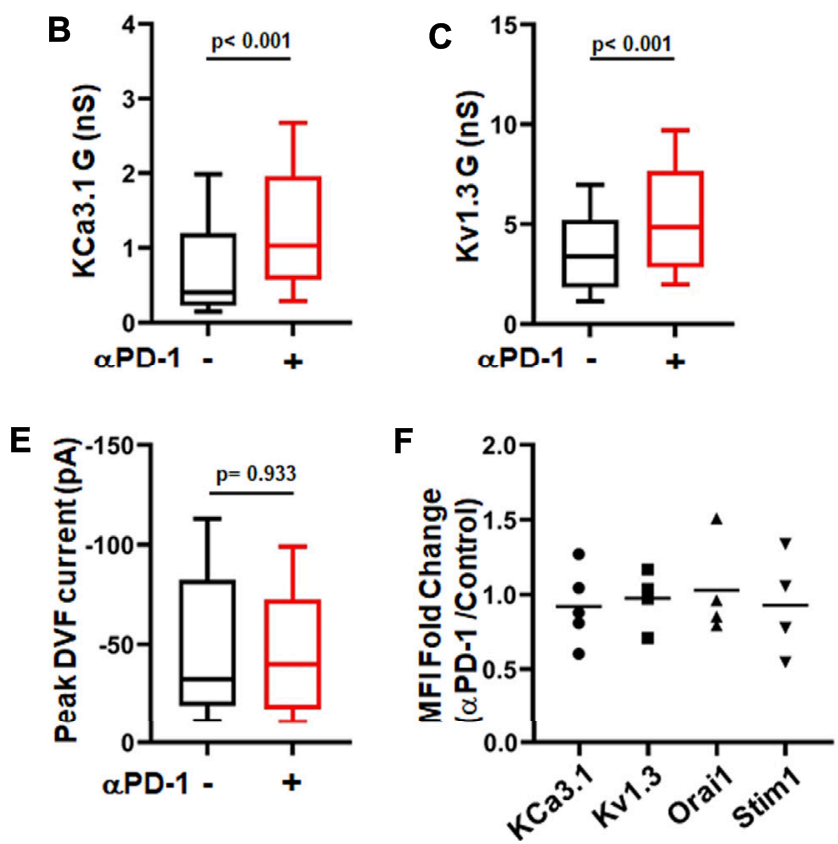

FIGURE 1 | aPD-1 treatment increases $\mathrm{K}^{+}$channel activity in HNSCC T cells. (A) Representative current traces of KCa3.1 and Kv1.3 channels recorded in wholecell mode of voltage-clamp configuration in activated CD8 ${ }^{+}$PBTs cells from a HNSCC patient in absence or presence of $a$ PD- 1 (10 $\mu \mathrm{g} / \mathrm{ml}$, for $6 \mathrm{~h}$ ). Data are normalized to maximum current at $+50 \mathrm{mV}$ recorded using a ramp pulse protocol from $-120 \mathrm{mV}$ to $+50 \mathrm{mV}$ for $200 \mathrm{~ms}$ every $15 \mathrm{~s}$. The holding potential used was $-70 \mathrm{mV}$. (B,C) KCa3.1 (B) and Kv1.3 (C) conductance (G) measured in the absence or presence of $\alpha$ PD -1 (10 $\mu \mathrm{g} / \mathrm{ml}, 6 \mathrm{~h}$ incubation) in CD8 ${ }^{+} \mathrm{PBTs}$ of HNSCC patients $(n=68$ cells without pembrolizumab and $n=55$ cells with pembrolizumab from 14 patients). (D) Representative current traces of divalent free current (DVF) through CRAC channels recorded in whole-cell mode of voltage-clamp configuration in activated CD8 ${ }^{+}$PBTs from a HNSCC patient. Data were recorded using a ramp pulse protocol from -100 to $+100 \mathrm{mV}$ with at holding potential of $+30 \mathrm{mV}$ every $1.5 \mathrm{~s}$. Cells were perfused with $0 \mathrm{mM} \mathrm{Ca}{ }^{2+}$ solution ( $1 \mathrm{~min}$ ) followed by $20 \mathrm{mM}$ Ca ${ }^{2+}$ ( $1 \mathrm{~min}$ ) and DVF solutions (2 min, see methods) to amplify currents during recordings. (E) Peak DVF current values measured in absence and presence of aPD-1 (10 $\mu \mathrm{g} / \mathrm{ml}$, $6 \mathrm{~h}$ incubation) in CD8 ${ }^{+}$PBTs of HNSCC patients ( $n=34$ cells without aPD-1 and $n=31$ cells with aPD- 1 from 8 patients). The values in panels (B,C) and (E) are represented as box plots: the horizontal line indicates the median; the lower box is the $25^{\text {th }}$ percentile; the upper box is the $75^{\text {th }}$ percentile; and the whiskers represent the $10^{\text {th }}$ and $90^{\text {th }}$ percentiles. (F) lon channel expression (KCa3.1, Kv1.3, Orai1 and STIM1) in HNSCC patient T cells after treatment with aPD-1 (10 $\mu$ g/ml for 6 h). Effect of aPD-1 treatment is shown as ratio of mean fluorescence intensity (MFI, fold change) values of treatment versus control group. Data are represented as scatter plot where each symbol represents an individual patient $(n=4-5)$. Horizontal line represents mean values for each group. Data in panels (B,C,E) were analyzed by Mann-Whitney rank sum test.

KCa3.1 and Kv1.3 activity in CD8 ${ }^{+}$PBTs (Newton et al., 2020). Herein, we conducted in vitro experiments to study in detail the effect of pembrolizumab on ion channels in HNSCC T cells. We tested the effect of a short exposure $(6 \mathrm{~h})$ to pembrolizumab ( $a$ PD- 1 antibody) $(10 \mu \mathrm{g} / \mathrm{ml})$ on activated CD8 ${ }^{+}$PBTs. Wholecell currents were measured before and after $\mathrm{aPD}-1$ treatment to assess KCa3.1 and Kv1.3 activity (Figure 1A). aPD-1 treatment increased the conductance of both KCa3.1 and Kv1.3 channels (Figures 1B,C). We did not detect any effect of $\alpha$ PD-1 on CRAC channel activity (Figures 1D,E). Further control experiments ruled out the possibility that the increase in KCa3.1 and Kv1.3 activity was an artefact due to the extra $6 \mathrm{~h}$ the cells treated with aPD-1 were maintained in culture. The KCa3.1 and Kv1.3 conductance in untreated cells at the beginning of the experiment $(0 \mathrm{~h})$ and after $6 \mathrm{~h}$ (equivalent to the time of exposure to $\alpha \mathrm{PD}-1$ ) were comparable (Supplementary Figure S1). There was no difference in the capacitance values, an arbitrary measure of cell size and activation state, before and after treatment with pembrolizumab (Supplementary Figure S2). Furthermore, flow cytometry experiments showed no changes in the expression of either ion channels or activation and exhaustion markers in $\mathrm{CD}^{+}$PBTs from HNSCC patients after aPD-1 treatment (Figure 1F; Supplementary Figures S3-S4). These findings indicate that $\alpha \mathrm{PD}-1$ treatment increases KCa3.1 and Kv1.3, but not CRAC channel, activity in $\mathrm{CD}^{+}$PBTs of HNSCC patients. Furthermore, aPD-1 treatment did not change the expression of typical markers associated with exhaustion and activation in $\mathrm{CD}^{+}$PBTs of HNSCC patients.

\section{PD-1 Blockade increases $\mathrm{Ca}^{2+}$ Fluxing Abilities in T Cells From a Subset of HNSCC Patients}

Since $\mathrm{K}^{+}$channels regulate $\mathrm{T}$ cell $\mathrm{Ca}^{2+}$ signaling and effector functions, we investigated the functional consequences of the increase in $\mathrm{K}^{+}$channel activity in $\mathrm{CD} 8^{+}$PBTs of HNSCC patients induced by $\alpha \mathrm{PD}-1$ treatment. We measured the $\mathrm{Ca}^{2+}$ fluxing abilities of $\mathrm{CD}^{+}$PBTs from HNSCC patients using a TG-based protocol that allows us to assess $\mathrm{Ca}^{2+}$ fluxes dependent on ion 

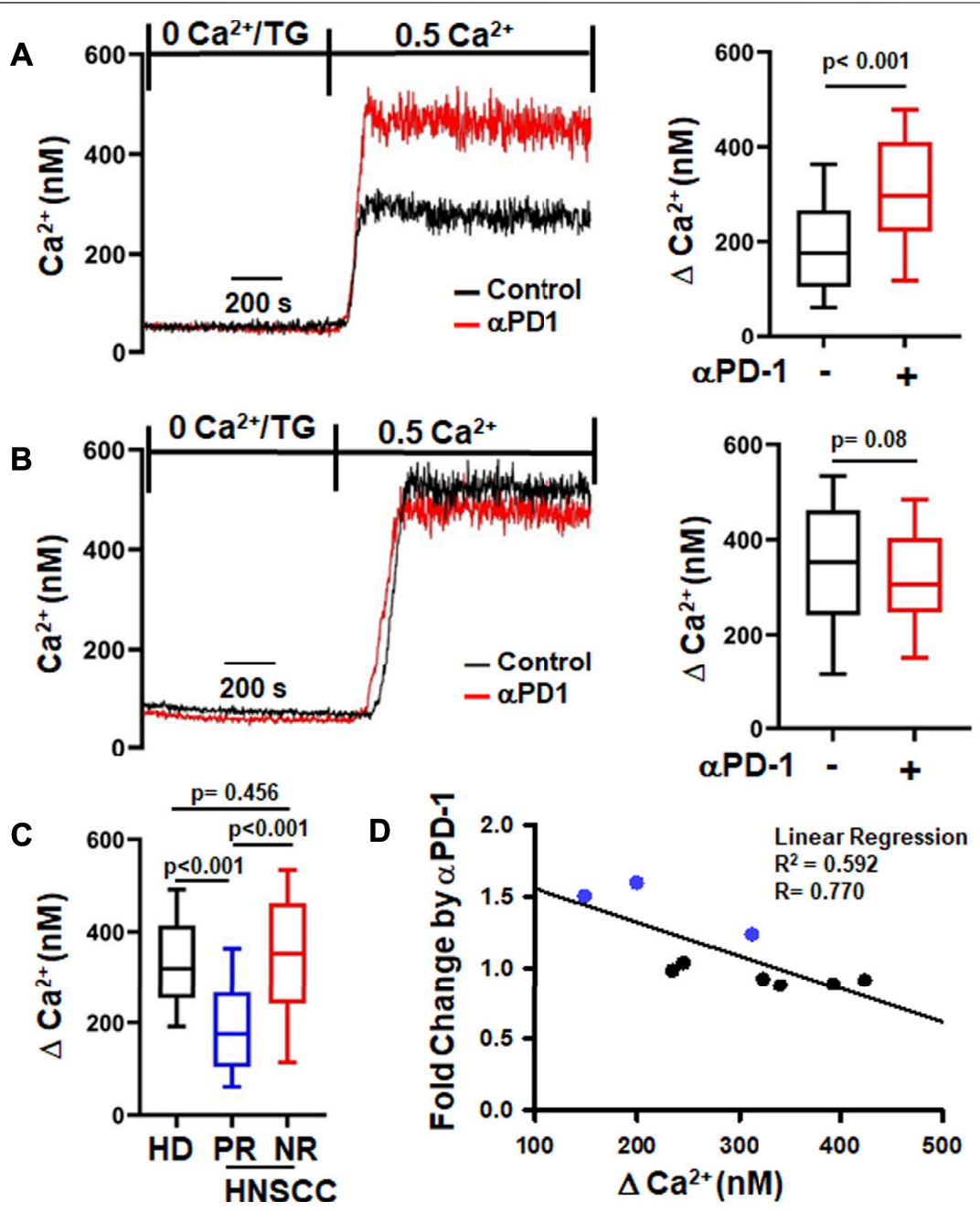

FIGURE 2 | aPD-1 increases $\mathrm{Ca}^{2+}$ fluxes in PBTs of a subset of HNSCC patients. (A,B) Representative intracellular Ca ${ }^{2+}$ recordings in activated CD8 ${ }^{+}$PBTs of HNSCC patients are shown on the left side. Cells loaded with Fura-2 were perfused with thapsigargin (TG) in $0 \mathrm{mM} \mathrm{Ca}^{2+}$. Perfusion with $0.5 \mathrm{mM}$ Ca ${ }^{2+}$ yields a rapid influx of $\mathrm{Ca}^{2+}$ (See Material and Methods). Two types of $\mathrm{Ca}^{2+}$ responses to $\mathrm{aPD}-1$ were observed. A significant increase in $\mathrm{Ca}{ }^{2+}$ after $\mathrm{aPD}-1$ (10 $\mu \mathrm{g} / \mathrm{ml}$ for $6 \mathrm{~h}$ ) was defined as positive response, $\mathrm{PR}$ (A, left) while no change in $\mathrm{Ca}^{2+}$ response was defined as no-response, NR (B, left). The subset of HNSCC patients $(n=3 / 9)$ showing positive $\mathrm{Ca}^{2+}$ response are reported in panel (A, right) while patients $(n=6 / 9)$ with no-response are shown in panel (B, right). The corresponding single cell $\Delta \mathrm{Ca}{ }^{2+}$ values (peak minus baseline before peak) measured in the absence and presence of $a$ PD-1 of PR and NR are shown in the right panels ( $n=82-99$ cells from 3 patients in PR and $n=140-149$ cells from 6 patients in NR). (C) Comparison of $\Delta \mathrm{Ca}^{2+}$ values of activated CD8 ${ }^{+}$PBTs from HDs $n=376$ cells from nine donors and PR ( $n=82$ cells from 3 patients) and NR ( $n=146$ cells from 6 patients) HNSCC patients at baseline (before aPD-1). The $\Delta \mathrm{Ca}^{2+}$ values in panels (A,B,C) are represented as box and whisker plots. The lower and upper bound of the box represent $25^{\text {th }}$ and $75^{\text {th }}$ percentiles respectively. Median values are shown as horizontal line in the box. The lower and upper error represents $10^{\text {th }}$ and $90^{\text {th }}$ percentile respectively. (D) Relationship between $\Delta \mathrm{Ca}^{2+}$ values before aPD-1 treatment and fold increase in $\Delta \mathrm{Ca}{ }^{2+}$ after treatment with aPD-1 antibody in CD8 ${ }^{+}$PBTs of HNSCC patients $(n=9)$ are represented as correlation analysis. Individual patients from PR and NR group are marked in blue (PR) and black (NR). Data for panel (A) were analyzed using unpaired student's $t$-test. Data for panel (B) were analyzed using Mann-Whitney rank-sum test. Data in panel $\mathrm{C}$ were analyzed using ANOVA $(p<0.001)$ followed by Dunn's test and data in panel D were analyzed using linear regression.

channel activity while bypassing the TCR (Newton et al., 2020). Activated $\mathrm{CD}^{+}$PBTs from HNSCC patients were treated with aPD-1 for $6 \mathrm{~h}$ (same protocol as Figure 1) and $\mathrm{Ca}^{2+}$ fluxes were measured. We observed variable $\mathrm{Ca}^{2+}$ responses in these patients' samples. Therefore, we defined the presence or absence of a response to $\alpha \mathrm{PD}-1$ based on a statistically significant increase in $\Delta \mathrm{Ca}^{2+}$ values before and after in vitro aPD-1 treatment in individual patients. In a subset of patients $(n=3 / 9)$, aPD-1 treatment increased the $\mathrm{Ca}^{2+}$ response of T cells (Figure $2 \mathbf{A}$ ). We termed these patients as "positive-response" (PR) patients. Patients whose $\mathrm{T}$ cells did not show any increase in the $\mathrm{Ca}^{2+}$ response to $\alpha \mathrm{PD}-1$ treatment in vitro were defined as "no-response" (NR) patients $(n=6 / 9)$, (Figure 2B). aPD-1 treatment induced a $46 \%$ increase in $\Delta \mathrm{Ca}^{2+}$ in the PR group (Figure 2A). Interestingly, we observed that the $\Delta \mathrm{Ca}^{2+}$ at baseline (before $\alpha \mathrm{PD}-1$ treatment) of $\mathrm{CD}^{+}$PBTs from PR patients was $67 \%$ lower than NR patients; even lower than that of HDs CD8 ${ }^{+}$ $\mathrm{PBTs}$ (Figure 2C). Indeed, we observed a significant negative 


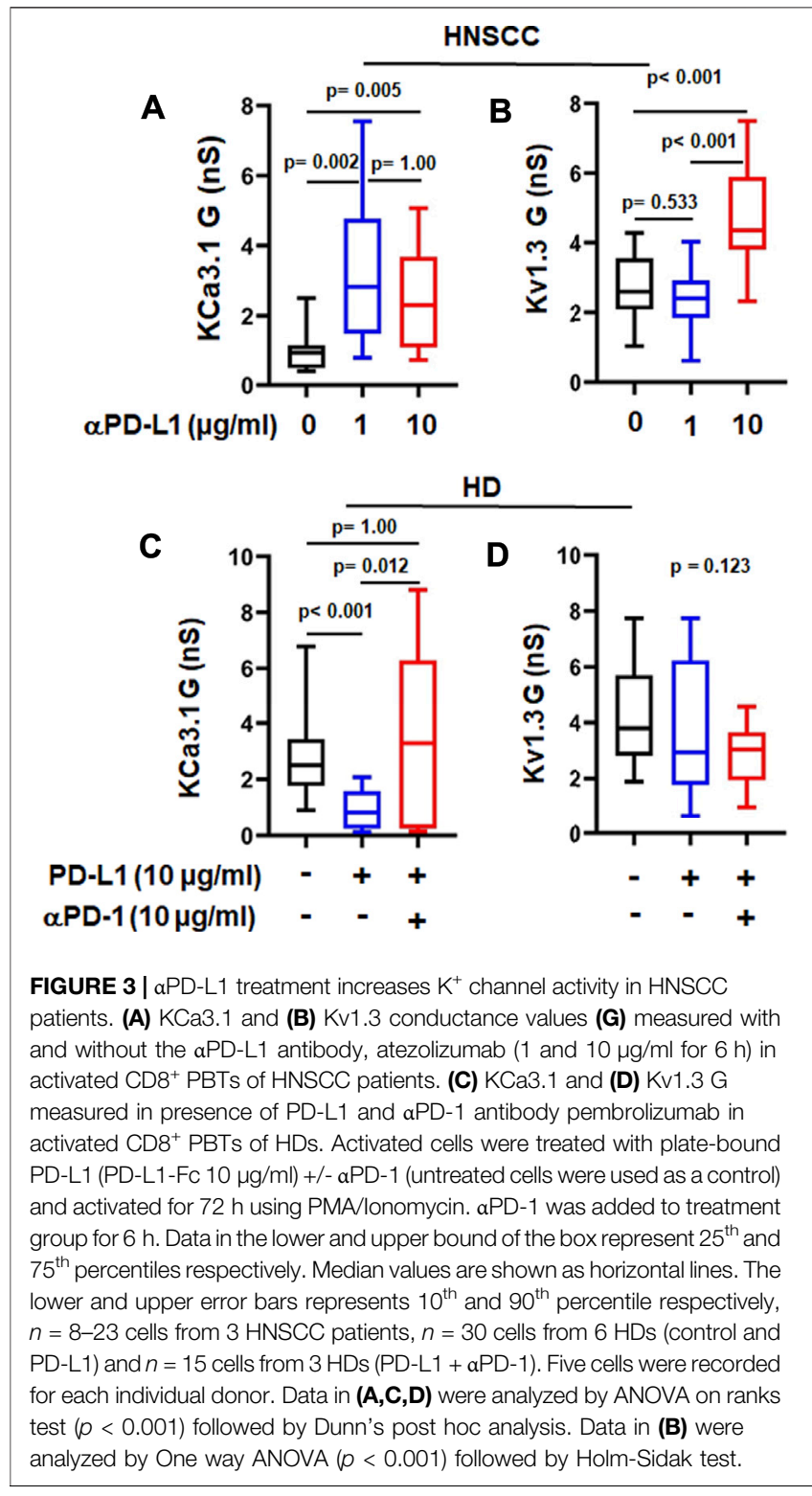

correlation between the increase in $\Delta \mathrm{Ca}^{2+}$ (fold change for individual patient) induced by $\alpha \mathrm{PD}-1$ versus the baseline $\Delta \mathrm{Ca}^{2+}$ of all HNSCC patients (Figure 2D). We performed this type of analysis because we wanted to assess whether the mean baseline $\Delta \mathrm{Ca}^{2+}$ of a patient could be a predictor of his/her ability to respond to pembrolizumab. This indicates that HNSCC patients whose $\mathrm{CD}^{+}$PBTs have low baseline $\mathrm{Ca}^{2+}$ fluxing abilities were more likely to show a positive response to aPD1 treatment. Overall, these results indicate that $\alpha \mathrm{PD}-1$ treatment has a rapid effect on ion channel-regulated $\mathrm{Ca}^{2+}$ fluxes of HNSCC $\mathrm{CD}^{+} \mathrm{PBT}$ and point to baseline $\mathrm{Ca}^{2+}$ fluxing ability of $\mathrm{T}$ cells as a possible predictive marker of $\alpha \mathrm{PD}-1$ treatment response. However, questions still remain about the mechanisms by which $a \mathrm{PD}-1$ antibody regulates $\mathrm{K}^{+}$channel activity: is this effect the result of blocking PD-L1/PD-1 binding, and what are the downstream signaling pathways involved?

\section{The Effect of aPD-1 Treatment on $\mathrm{K}^{+}$ Channels is due to the Disruption of PD-L1/ PD-1 Binding}

We tested the corollary of our earlier observation with aPD-1 treatment hypothesizing that prevention of or breaking the interaction of PD-L1 with PD-1 is responsible for the improved $\mathrm{K}^{+}$channel function. While we have no cancer cells in our in vitro setting, $\mathrm{CD}^{+}$PBTs of HDs and HNSCC patients can be a source of PD-L1 as both express it (Supplementary Figure S5). Others have shown that human $\mathrm{CD}^{+}{ }^{+}$T cells express PD-L1 (Succaria et al., 2020). Therefore, we next tested the effect of an anti-PD-L1 antibody ( $\alpha \mathrm{PD}$ L1), atezolizumab, on KCa3.1 and Kv1.3 channels in activated CD8 ${ }^{+}$ PBTs of HNSCC patients reasoning that if the effect of pembrolizumab is due to the disruption of PD-L1/PD-1 binding, a similar effect should be produced by $\alpha \mathrm{PD}-\mathrm{L} 1$. Short-term treatment of HNSCC T cells with atezolizumab ( 1 and $10 \mu \mathrm{g} / \mathrm{ml}$ for $6 \mathrm{~h}$ ) increased KCa3.1 function, while Kv1.3 function was increased only at the higher concentration (Figures 3A,B). These results suggest that engagement of PD-L1 by PD-1 may have negative effects on $\mathrm{K}^{+}$channel function in HNSCC T cells and disruption of its binding to the cognate receptor restores the channel's function. Therefore, we tested the effect of PD-L1 (plate-bound PD-L1-Fc; $10 \mu \mathrm{g} / \mathrm{ml}$ ) on KCa3.1 and Kv1.3 in CD8 ${ }^{+}$PBTs of HDs. Cells were incubated with PD-L1 for $72 \mathrm{~h}$ followed by treatment with aPD-1 antibody $(10 \mu \mathrm{g} / \mathrm{ml})$ for $6 \mathrm{~h}$. Our data showed that PD-L1 reduced KCa3.1 activity by $65.44 \%$, but, at this concentration, had no effect on Kv1.3 (Figures 3C,D). A similar effect was observed after 5 days exposure to PD-L1 (Supplementary Figure S6). Furthermore, aPD1 treatment reversed the effect of PD-L1 on KCa3.1 activity (Figure 3C). These results show that, in $\mathrm{HDs}_{\mathrm{CD}}{ }^{+} \mathrm{PBTs}$, KCa3.1 channels appear highly sensitive to PD-1 signaling, more than Kv1.3.

We then measured $\mathrm{Ca}^{2+}$ fluxes in activated $\mathrm{CD}^{+}{ }^{+} \mathrm{PBT}$ s cells of HDs exposed to PD-L1 to assess the functional effect of PD-L1. Nine out of twelve samples from HDs showed response to PD-L1 and only the $\mathrm{CD} 8^{+}$PBTs samples from donors that showed this response were used in analysis. Cells exposed to PD-L1 showed a small $(14 \%)$ decrease in $\Delta \mathrm{Ca}^{2+}$ as compared to control cells (Figure 4B). Treatment with pembrolizumab significantly increased $\Delta \mathrm{Ca}^{2+}$ by $39 \%$ in PD-L1 exposed cells, and by $25 \%$ in $9 / 12 \mathrm{HDs}$ in the control untreated group (Figures $\mathbf{4 A , B}$ ). It is noteworthy that treatment with pembrolizumab showed a similar increase of $45 \%$ in $\triangle \mathrm{Ca}^{2+}$ in HNSCC CD8 ${ }^{+}$PBTs (Figure 2A). These results indicate that $\mathrm{PD}-\mathrm{L} 1$ reduced $\mathrm{Ca}^{2+}$ fluxing abilities of $\mathrm{T}$ cells in HDs thus supporting the notion that high exposure to PD-L1 may decrease the $\mathrm{Ca}^{2+}$ fluxing ability of HNSCC $\mathrm{CD}^{+}$ PBTs like it occurs for TILs (Chimote et al., 2017). Furthermore, they suggest that KCa3.1 may be highly sensitive to PD-1 signaling, more than Kv1.3. We thus proceeded to investigate the mechanism through which $\mathrm{PD}-1$ regulates KCa3.1 activity.

\section{PI3K Signaling and CaM Mediate PD-L1 inhibition of KCa3.1 Channels}

Activation and function of KCa3.1 is controlled by a variety of molecules and phosphorylation events (Ohya and Kito, 2018). 

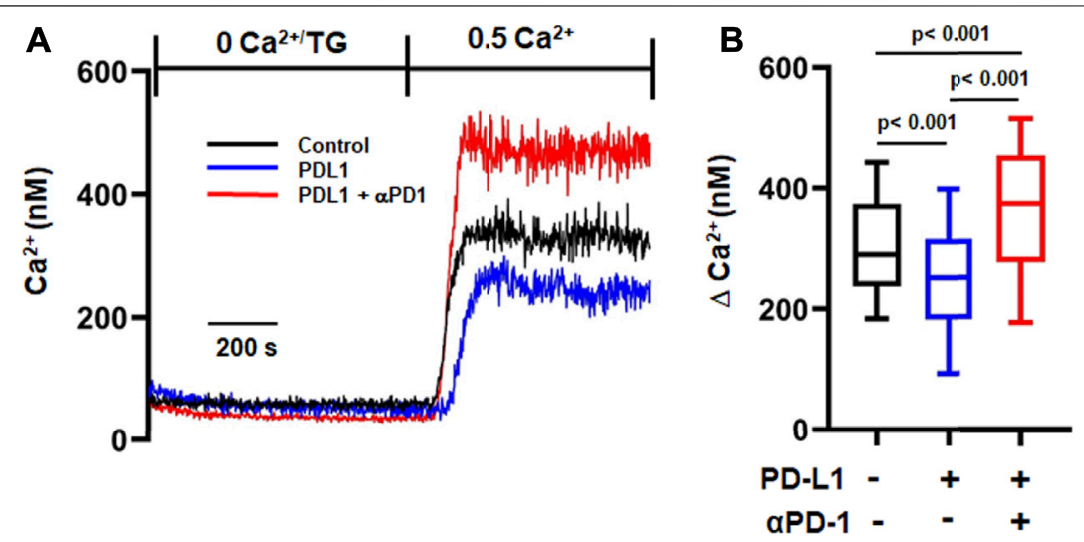

FIGURE 4 | aPD-1 rescues the reduced $\mathrm{Ca}^{2+}$ influx induced by PDL-1-FC in HDs. (A) Representative traces of intracellular Ca ${ }^{2+}$ response in activated CD8 ${ }^{+}$PBTs

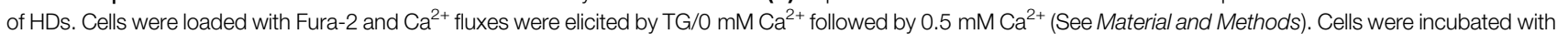
plate bound PD-L1 (PD-L1-Fc, $10 \mu \mathrm{g} / \mathrm{ml}$ for $72 \mathrm{~h}$ ) followed by treatment with aPD-1 (10 $\mu \mathrm{g} / \mathrm{ml}$ for $6 \mathrm{~h}$ ). (B) Summary of $\Delta \mathrm{Ca}^{2+}$ values of activated CD8 ${ }^{+}$PBTs of HDs ( $n=160-255$ cells from nine HDs, 20-50 cells were recorded from a single HD, see Methods). The values in panel B are represented as box and whisker plots. The lower and upper bound of the box represent $25^{\text {th }}$ and $75^{\text {th }}$ percentiles respectively. Median values are shown as horizontal line. The lower and upper error bars represents $10^{\text {th }}$ and $90^{\text {th }}$ percentile respectively. Data were analyzed using ANOVA $(p<0.001)$ on ranks followed by Dunn's post hoc analysis.

KCa3.1 function requires binding of the intracellular $\mathrm{Ca}^{2+}$ sensor calmodulin (CaM). We recently showed that $\mathrm{CD}^{+}$PBTs of HNSCC patients have reduced levels of CaM which diminished their KCa3.1 activity (Chimote et al., 2020). PD-L1 is available in the plasma of HNSCC patients both as soluble molecule or carried by exosomes, and the presence of PD-L1 ${ }^{+}$ exosomes has been correlated with the increased immune suppressive state of these patients (Theodoraki et al., 2018). These findings raise the possibility that a reduction in CaM by PD-L1 mediates the suppression of KCa3.1 currents. Therefore, we proceeded to determine the signaling pathways involved in PD-L1/PD-1 signaling by exposing HD T cells to PD-L1 for 3 days ( $72 \mathrm{~h}$; like in the experiments reported above) and 5 days. The latter resembles more the HNSCC patients' setting where $\mathrm{T}$ cells are exposed to PD-L1, both in circulation and in the tumor, for a long time. Exposure to PD-L1 for 3 days did not reduce $\mathrm{CaM}$ expression (Figures $\mathbf{5 A}, \mathbf{B}$ ) and, consequently, intracellular supplementation of $\mathrm{CaM}$ did not rescue $\mathrm{KCa} 3.1$ inhibition (Figures 5C,D). We thus investigated whether the phosphoinositide 3-kinase (PI3K)-phosphatidylinositol-3 phosphatase (PI3P) signaling pathway was instead involved. In $\mathrm{T}$ lymphocytes, PI3K favors the production of $\mathrm{PI} 3 \mathrm{P}$ from phosphatidylinositol (PI); PI3P activates the nucleoside diphosphate kinase B (NDPK-B) which, ultimately, increases KCa3.1 activity via histidine phosphorylation (Srivastava et al., 2005; Srivastava et al., 2006b). PD-1 signaling in T cells involves the blockade of PI3K and, consequently, the suppression of downstream effector functions (Patsoukis et al., 2013). We performed patch-clamp experiments to determine if the inhibition of KCa3.1 activity by PD-L1 can be mimicked by the PI3K inhibitor LY294002 and rescued by PI3P. Activated $\mathrm{CD}^{+}$PBTs from HDs were pretreated with LY294002 $(10 \mu \mathrm{M}$, for $15 \mathrm{~min}$ ) followed by measurement of $\mathrm{KCa} 3.1$ in presence and absence of PI3P (100 nM) delivered intracellularly via patch pipette (Figure 6A). LY294002, similar to PD-L1, reduced
KCa3.1 currents while PI3P reversed this inhibition in both LY294002 and PD-L1 treated cells. (Figures 6A-C). These results provide the evidence of involvement of $\mathrm{PI} 3 \mathrm{~K}$ in the early (3 days) effect of PD-L1 on KCa3.1 activity. Longer exposure to $\mathrm{PD}-\mathrm{L} 1$ revealed a diminished contribution of $\mathrm{PI} 3 \mathrm{~K}$ signaling and a role for CaM becomes evident (Figures 6D,E). Electrophysiological experiments showed that PI3P only partially restored the KCa3.1 activity of $\mathrm{HDs} \mathrm{CD}^{+} \mathrm{PBT}$ s treated with PDL1 for 5 days (Figure 6D). Flow-cytometry data showed that at this time point there was a significance $40 \%$ reduction in CaM expression and not KCa3.1. Interestingly, we observed also a selective reduction in Stim1 (the partner protein of Orail that forms CRAC channels) (Figure 6E). Overall, these findings support a role for PI3P signaling and $\mathrm{CaM}$ in mediating the effect of PD-L1/PD-1 interaction on KCa3.1 channels in $\mathrm{CD}^{+}$ T cells.

\section{DISCUSSION}

Resistance to immunotherapy in cancer is attributed to a weak or blunted immune response. Engagement of the immune checkpoint receptor PD-1 by its ligand PD-L1 is part of the immune escape mechanism of cancer cells (Farhood et al., 2019). Herein, we provide evidence of a role for $\mathrm{K}^{+}$channels as early regulators of $\mathrm{PD}-\mathrm{L} 1 / \mathrm{PD}-1$ mediated suppression of cytotoxic $\mathrm{T}$ cells in HNSCC patients.

It is well established that blockade of PD-L1/PD-1 interaction increases $\mathrm{T}$ cell function and antitumor immunity (Alsaab et al., 2017). In this study, we report that disruption of PD-L1/PD-1 binding by aPD-1 and aPD-L1 antibodies, rapidly (within $6 \mathrm{~h}$ ) increased KCa3.1 and Kv1.3 activities in $\mathrm{CD}^{+}$PBTs of HNSCC patients. Studies in murine models and human subjects with solid malignancies show that $\alpha \mathrm{PD}-1$ treatment improves $\mathrm{T}$ cell activation as indicated by the increased expression of 

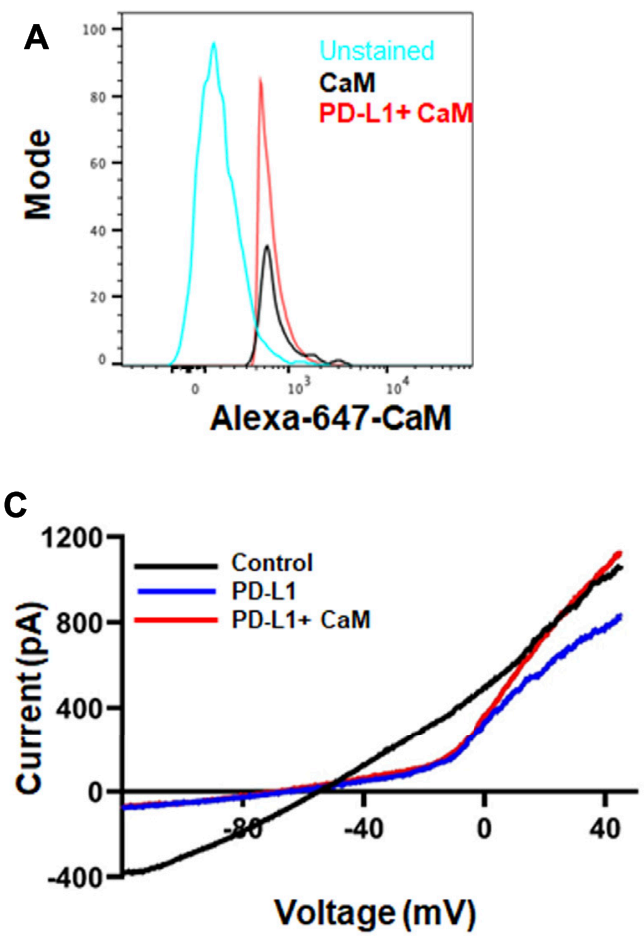
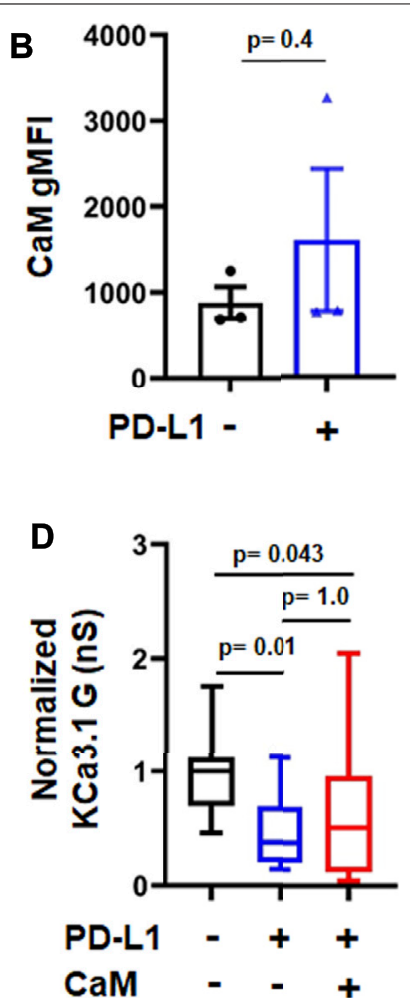

FIGURE 5 | Short-time treatment with PD-L1 decreases KCa3.1 activity in a calmodulin-independent manner. (A) Flow cytometry histogram and geometric mean fluorescence intensity (gMFI) values (B) for CaM expression in activated CD8 ${ }^{+}$PBTs from HD donors $(n=3)$ in the absence and presence of PD-L1. (C) Representative recordings of KCa3.1 currents in activated CD8 ${ }^{+}$PBTs from HDs showing the effect of PD-L1 (PD-L1-FC, $10 \mu \mathrm{g} / \mathrm{ml}$ ) and CaM (50 $\mu M$ ). (D) Average normalized KCa3.1 conductance $(\mathbf{G}, \mathrm{nS})$ measured in the absence and presence of PD-L1, with and without CaM. All conductance values are normalized to average conductance value obtained from control recordings. Cells were pre-incubated with plate-bound PD-L1 (PD-L1-Fc,10 $\mu \mathrm{g} / \mathrm{ml}$, for 72 h) activated using anti-CD3/CD28 antibodies and treated with or without $\mathrm{CaM}(50 \mu \mathrm{M})$, that was delivered intracellularly via patch pipette during recordings $(n=15-18 \mathrm{cells}$ per group from $3 \mathrm{HDs})$. The values in panel (B) are represented as bar graphs. Each symbol represent an individual HD. The values are represented as mean \pm SEM. The values in panel (D) are represented as box and whisker plots. The lower and upper bound of the box represent $25^{\text {th }}$ and $75^{\text {th }}$ percentiles respectively. Median values are shown as horizontal line. The lower and upper error bars represents $10^{\text {th }}$ and $90^{\text {th }}$ percentile respectively. Data in panel (B) were analyzed by $t$-test and data in panel (D) were analyzed by ANOVA on ranks $(p=0.008)$ with Dunn's post hoc analysis.

activation markers (Peng et al., 2012; Ascierto et al., 2019). Herein, we showed that short treatment with aPD-1 antibody (pembrolizumab) was not sufficient to change the expression of the $\mathrm{T}$ cell activation marker CD69, and yet produced changes in the activity of $\mathrm{K}^{+}$channels. These data suggest that activation of $\mathrm{K}^{+}$channels may be among the earliest changes produced by interruption of PD-1 signaling in $\mathrm{CD}^{+}$PBTs of HNSCC patients. We also showed that $\alpha \mathrm{PD}-1$ treatment affected exclusively $\mathrm{K}^{+}$ channels as it did not alter CRAC channels. Furthermore, the effect of $\alpha$ PD- 1 antibody on $\mathrm{K}^{+}$channels could not be ascribed to changes in channel protein expression or activation/exhaustion state of T cells as markers of T cell activation and exhaustion and ion channel proteins were all unchanged. This is in agreement with what we observed in T cells from HNSCC patients treated with pembrolizumab (Newton et al., 2020).

A well-established functional consequence of increased KCa3.1 and Kv1.3 activities in $\mathrm{T}$ cells is an increase in $\mathrm{Ca}^{2+}$ fluxing abilities that is necessary for cytokine production and cytotoxicity (Feske et al., 2012). In the present study, we measured TCR-independent $\mathrm{Ca}^{2+}$ fluxes to assess the consequences of the positive effect of $\alpha \mathrm{PD}-1$ treatment on $\mathrm{K}^{+}$channel activity. Indeed, we observed that pembrolizumab produces a rapid increase in $\mathrm{Ca}^{2+}$ fluxing abilities of $\mathrm{CD}^{+}$PBTs in a subset of HNSCC patients. The in vitro positive $\mathrm{Ca}^{2+}$ response to pembrolizumab was observed in $33 \%$ of patients. This response is in line with the objective response reported in a recent clinical trial (KEYNOTE-048) in HNSCC patients (Burtness et al., 2019; Kok, 2020). We also observed that a low baseline $\mathrm{Ca}^{2+}$ fluxing ability of $\mathrm{CD} 8^{+} \mathrm{PBT}$ from HNSCC patients predicts the response to pembrolizumab. It is possible that HNSCC patients whose $\mathrm{T}$ cells display lower $\mathrm{Ca}^{2+}$ fluxing abilities in vitro had a higher exposure to circulating PD-L1 (either soluble or via tumor-derived exosomes) in vivo, and they will ultimately benefit the most from $\alpha$ PD-L1 and $\alpha$ PD-1 blockade. An in vitro study showed that PD-L1 decreases TCR dependent $\mathrm{Ca}^{2+}$ fluxes in a dose dependent manner in human $\mathrm{T}$ cells overexpressing the $\mathrm{PD}-1$ receptor (Wei et al., 2013). In agreement with this study, we reported here that PD-L1 inhibits ion channel-dependent $\mathrm{Ca}^{2+}$ fluxes in $\mathrm{CD}^{+} \mathrm{T}$ cells. Furthermore, multiple studies have shown that a high PD-L1 expression in 

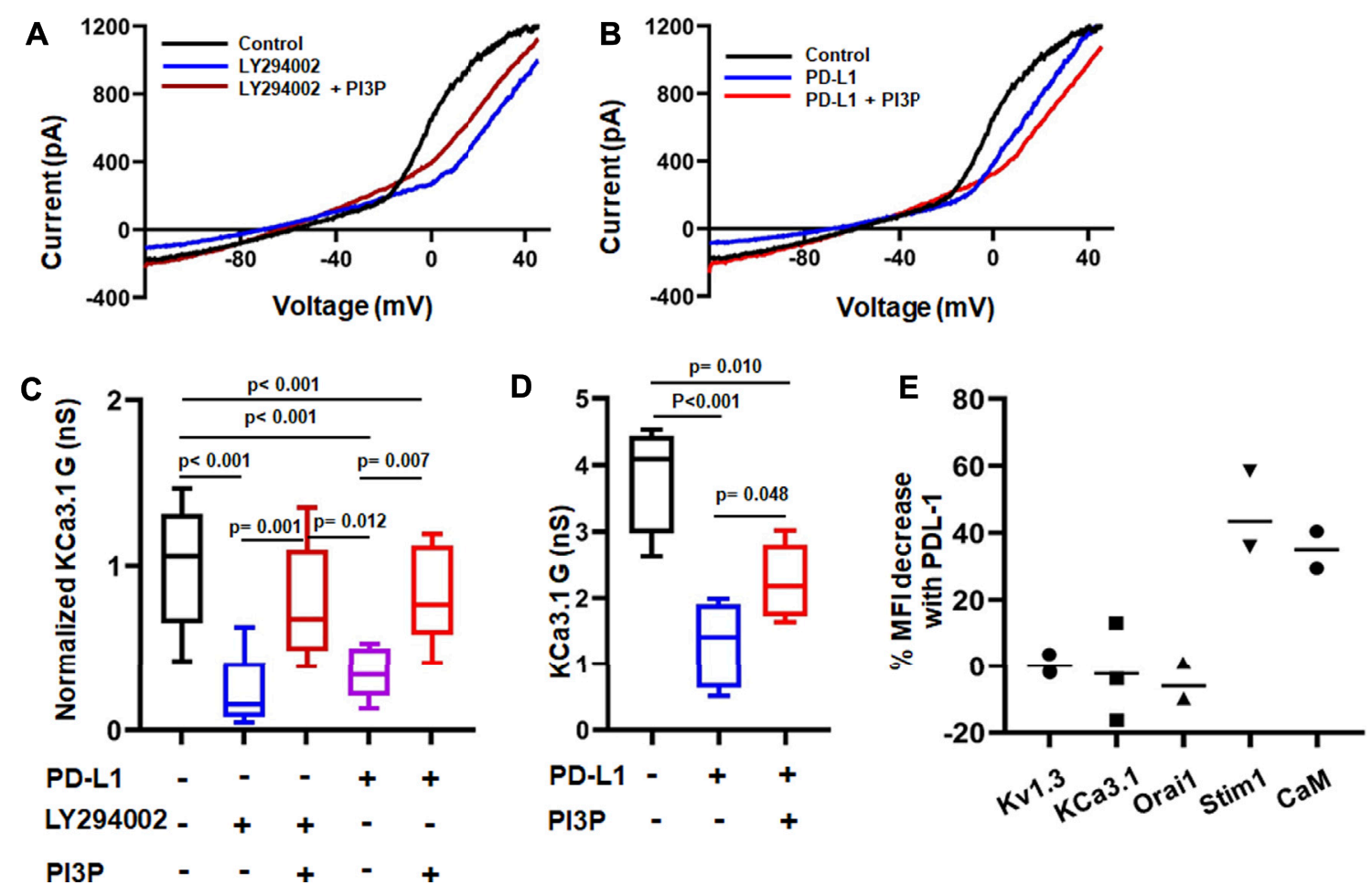

FIGURE 6 | Differential time-dependent involvement of PI3K and calmodulin on PD-L1 mediated inhibition of KCa3.1 channels. (A,B) Representative recordings of KCa3.1 channels in activated CD8 ${ }^{+}$PBTs from HDs showing the effect of the PI3K inhibitor LY294002 (10 $\left.\mu \mathrm{M}\right)+/$ - phosphatidylinositol-3 phosphatase (PI3P) (100 nM) (A) and PD-L1 (PD-L1-Fc, 10 fg/ml) +/- PI3P (100 nM) (B). (C) Summary of the pharmacological modulation of KCa3.1 channels byLY294002 and PI3P in the absence and presence of plate bound PD-L1 in activated CD8 ${ }^{+}$PBTs of HDs. Cells were activated using anti-CD3/CD28 antibodies for $72 \mathrm{~h}$. Cells were perfused with LY294002 for 15 min followed by patch clamp recordings with and without PI3P, delivered intracellularly via patch pipette ( $n=$ eight to nine cells per group from 3 HDs). All KCa3.1 conductance (G) values are normalized to the average G of the control group (drug-free). (D) KCa3.1 G measured in absence or presence of PD-L1 in activated CD8 ${ }^{+}$PBTs of HDs. Cells were treated with plate-bound PD-L1 (PD-L1-Fc, $10 \mu \mathrm{g} / \mathrm{ml}$ ) and activated using anti-CD3/CD28 antibodies for $120 \mathrm{~h}$ PI3P was delivered intracellularly via the patch pipette during the electrophysiological experiments (drug-free control). Cells were held at $-70 \mathrm{mV}, n=$ four to five cells per group from one HD. The values in panel (C,D) are represented as box and whiskers plot. The lower and upper bound of the box represent $25^{\text {th }}$ and $75^{\text {th }}$ percentiles respectively. Median values are shown as horizontal line. The lower and upper error bars represents $10^{\text {th }}$ and $90^{\text {th }}$ percentile respectively. (E) Percentage change in mean fluorescence intensity (MFI) of ion channels (Kv1.3, KCa3.1, Orai1, Stim1) and Calmodulin (CaM) measured using flow cytometry. Each dot represents an individual HD and the horizontal black line represents the mean value. Data in panel (C) were analyzed by One Way ANOVA $(p<0.001)$ followed by Holm-Sidak's post hoc analysis. Data in (D) were analyzed by One way ANOVA followed by Holm-Sidak's post hoc analysis.

cancer cells correlate with a good response to aPD-1/aPDL-1 blocking antibodies (Evrard et al., 2020).

Our data showed that while KCa3.1 and Kv1.3 channels mediate the response to $\alpha \mathrm{PD}-1$ and $\alpha \mathrm{PD}-\mathrm{L} 1$ antibodies, there may be a difference in sensitivity. Indeed, a higher concentration of aPD-L1 was necessary to unleash Kv1.3 activity in HNSCC $\mathrm{T}$ cells. Furthermore, the concentration of PD-L1 activating antibodies (PD-L1-Fc) we used $(10 \mu \mathrm{g} / \mathrm{ml})$ suppressed $\mathrm{KCa} 3.1$, but not Kv1.3, currents in $\mathrm{CD}^{+}$PBTs of HDs, and, in this setting, aPD-1 antibody increased KCa3.1, but not Kv1.3, conductance. It is possible that inhibition of Kv1.3 requires higher concentrations of PDL-1 and/or a longer exposure. Our earlier observations in vivo in HNSCC patients treated with pembrolizumab showed increases KCa3.1, but not Kv1.3, channels activity in $\mathrm{CD}^{+} \mathrm{PBT}$ shortly after treatment (Newton et al., 2020). The effect of PD-1 blockade on $\mathrm{Kv} 1.3$ is only evident a long time after pembrolizumab administration. However, it is also possible that higher levels of PD-L1 may be needed for Kv1.3 inhibition. This could explain what we observed untreated
HNSCC patients (Chimote et al., 2018; Newton et al., 2020). We reported low KCa3.1 activity in $\mathrm{CD}^{+} \mathrm{PBTs}$, which is further reduced in TILs (Chimote et al., 2018; Newton et al., 2020). However, while we did not observe any reduction in Kv1.3 activity in HNSCC circulating $\mathrm{T}$ cells, there was a profound suppression of this channel activity/expression in TILs, particularly those in close proximity with cancer cells, (Chimote et al., 2018; Newton et al., 2020) where the levels of PD-L1 are higher than in circulation. Secreted PD-L1 is detected in the plasma of HNSCC patients contributing to reduced T cell function (Zhou et al., 2020). While we showed that CD8 ${ }^{+}$PBTs express PD-L1, we do not know the PD-L1 levels in our cell cultures and how they compare with those of PD-L1-Fc used in our experiments. The presence of $\mathrm{T}$ cell generated PD-L1 (endogenous PD-L1) explains the response to $\alpha \mathrm{PD}-1$ and aPD-L1 antibodies without pre-treatment with PD-L1-Fc (Figures 1, 3). It is very likely that PD-L1-Fc is in addition to the endogenous PD-L1. This explains why aPD-1 brings the $\mathrm{Ca}^{2+}$ response of $\mathrm{PD}-\mathrm{L} 1-\mathrm{Fc}$ treated cells to levels higher than 
"untreated" cells that are exposed to the endogenous PD-L1 (Figure 4B).

The high sensitivity of KCa3.1 to PD-L1 may be explained by the mechanisms by which $\mathrm{PD}-1$ regulates this channel which imply a fast kinase-mediated signaling event. We observed that the response of KCa3.1 channels to PD-1 ligation is mediated by PI3K-PI3P signaling and that this is the earliest pathway engaged. CaM downregulation follows in time. In $\mathrm{T}$ cells, activation of TCR signaling cascade leads to activation of PI3K/Akt [phosphatidylinositol 3-kinase (PI3K) and Akt/Protein Kinase $\mathrm{B})$ ], while ligation of $\mathrm{PD}-1$ results in a decrease in TCR proximal signaling through inhibition of PI3K activity (Seidel et al., 2018). $\mathrm{PI} 3 \mathrm{~K}$ has also been implicated in $\mathrm{KCa} 3.1$ regulation (Ohya and Kito, 2018). KCa3.1 channels have a histidine residue (H358) in the carboxy-terminus whose phosphorylation is regulated by PI3K, PI3P and NDPK-B kinase (Srivastava et al., 2006b; Srivastava et al., 2009; Srivastava et al., 2016). KCa3.1 channels in healthy human $\mathrm{CD} 4^{+} \mathrm{T}$ cells are inhibited by the non-selective PI3K blocker LY294002, while PI3P (whose formation from PI is facilitated by $\mathrm{PI} 3 \mathrm{~K}$ ) reverses this inhibition (Srivastava et al., 2006a). NDPK-B (the enzyme mediating PI3P effect on KCa3.1) knock-down decreases KCa3.1 without affecting the activity of Kv1.3 (Srivastava et al., 2006b). Similarly, in this study we showed that PD-L1-Fc at the concentration we used only inhibited KCa3.1, that this effect was reproduced by LY294002, and reversed by $\mathrm{PI} 3 \mathrm{P}$. While the $\mathrm{PI}$ K/PI3P signaling pathway is the dominant regulatory mechanism early upon $\mathrm{PD}-1$ ligation, a reduction in $\mathrm{CaM}$ expression contributes to reduced $\mathrm{KCa} 3.1$ activity later on. CaM serves as binding partner and intracellular $\mathrm{Ca}^{2+}$ sensor for KCa3.1 allowing the activation of this $\mathrm{Ca}^{2+}$-dependent channel (Cahalan and Chandy, 2009; Sforna et al., 2018). We have recently reported that membrane CaM levels are downregulated in activated $\mathrm{CD}^{+} \mathrm{T}$ cells of HNSCC patients (Chimote et al., 2020). In this study, the long-term effect of $\mathrm{PD}-1$ ligation in healthy $\mathrm{CD}^{+} \mathrm{T}$ cells is a decrease in CaM expression. CaM downregulation is not contributing to $\mathrm{KCa} 3.1$ suppression at earlier time points as indicated by the lack of effect of intracellular supplementation of CaM on KCa3.1 channels in PBTs of HDs treated for $72 \mathrm{~h}$ with PD-L1-Fc. At this stage, it is not clear if there is a correlation between PI3K and CaM. However, it is possible that the decrease in $\mathrm{Ca}^{2+}$ fluxing abilities that follows the reduction in KCa3.1 by PI3K inhibition may lead to reduced CaM. It has been reported that CaM gene expression is regulated by $\mathrm{Ca}^{2+}$ (Bosch et al., 1994).

Overall, our in vitro studies showed that $\alpha \mathrm{PD}-1$ induces an increase in $\mathrm{Kv} 1.3, \mathrm{KCa} 3.1$ and $\mathrm{Ca}^{2+}$ fluxing that ultimately will lead to improved anti-tumor response. $\mathrm{Ca}^{2+}$ signaling in fact regulates multiple effector functions of $\mathrm{T}$ cells, including the production of cytokines like IL- 2 and IFN- $\gamma$, and cytotoxicity (Feske et al., 2003). These are highly desirable anti-tumor functions. In agreement with the studies presented here, we have recently reported that in vivo administration of $\alpha \mathrm{PD}-1$ blockade followed by surgical resection increases $\mathrm{Ca}^{2+}$ fluxing abilities of $\mathrm{CD}^{+} \mathrm{T}$ cells by increasing the KCa3.1 and $\mathrm{Kv} 1.3$ channel function in head and neck cancer patients (Newton et al., 2020). Furthermore, a recent report on combination of immune checkpoint inhibitors, anti-PD-1 and anti-LAG-3 antibodies, showed significant increase in peak $\mathrm{Ca}^{2+}$ levels of $\mathrm{T}$ cells resulting in increased cytotoxicity (Sullivan et al., 2020).

In conclusion, the data presented in this manuscript highlight the crucial role of $\mathrm{K}^{+}$channels as early modulators of PD-1 signaling and point to them as therapeutic targets in restoring anti-tumor immunity in HNSCC patients. This study paves the way for further investigations about new therapeutic strategies in cancer that incorporate $\mathrm{K}^{+}$channel activators along with immune checkpoint inhibitors.

\section{DATA AVAILABILITY STATEMENT}

The original contributions presented in the study are included in the article/Supplementary Material, further inquiries can be directed to the corresponding author/s.

\section{ETHICS STATEMENT}

The studies involving human participants were reviewed and approved by University of Cincinnati Institutional Review Board (IRB no. 2014-4755). The patients/participants provided their written informed consent to participate in this study.

\section{AUTHOR CONTRIBUTIONS}

Conceptualization and design: LC, and VG Development of Methodology: VG, AC and MF-G, Formal analysis: VG, and AC Investigation: VG, AC, HN, MC, and MF-G. Resources: MC, MF-G, and HN. Writing (original draft): VG, and LC. Writing (review and editing): VG, AC, and LC Visualization: VG, AC, and MF-G Supervision: LC Project administration: VG, EJ, TW-D, and LC. Funding acquisition: LC, EJ, and TW-D. All authors discussed the results and commented on the manuscript.

\section{FUNDING}

This work was supported by the Office of the Assistant Secretary of Defense for Health Affairs, through the Peer Reviewed Cancer Research Program, under Award No W81XWH-17-1-0374 (LC, TW-D, and EJ). Opinions, interpretations, conclusions and recommendations are those of the authors and are not necessarily endorsed by the Department of Defense. The United States Army Medical Research Acquisition Activity, Fort Detrick, Maryland is the awarding and administering acquisition office. Additional funding was provided by the National Institutes of Health (grant R01-CA95286) to LC. HN was supported by NIH training grant T32 CA117846.

\section{ACKNOWLEDGMENTS}

We would like to acknowledge the clinical coordinators (Bethany Fuhrman and Shireen Desai) for their assistance in patient sample collection (Clinical Trials Office, University of Cincinnati Cancer 
Center). All flow cytometry data were acquired using equipment maintained by the Research Flow Cytometry Core (Division of Rheumatology) at Cincinnati Children's Hospital Medical Center and Shriners Hospital for Children Flow Cytometry Core, Cincinnati, $\mathrm{OH}$.

\section{REFERENCES}

Alsaab, H. O., Sau, S., Alzhrani, R., Tatiparti, K., Bhise, K., Kashaw, S. K., et al. (2017). PD-1 and PD-L1 Checkpoint Signaling Inhibition for Cancer Immunotherapy: Mechanism, Combinations, and Clinical Outcome. Front. Pharmacol. 8, 561. doi:10.3389/fphar.2017.00561

Ascierto, P. A., Bifulco, C., Buonaguro, L., Emens, L. A., Ferris, R. L., Fox, B. A., et al. (2019). Perspectives in Immunotherapy: Meeting Report from the "Immunotherapy Bridge 2018" (28-29 November, 2018, Naples, Italy). J. Immunother. Cancer 7 (1), 332. doi:10.1186/s40425-019-0798-3

Bauml, J. M., Aggarwal, C., and Cohen, R. B. (2019). Immunotherapy for Head and Neck Cancer: where Are We Now and where Are We Going? Ann. Transl. Med. 7 (Suppl. 3), S75. doi:10.21037/atm.2019.03.58

Bosch, M., López-Girona, A., Bachs, O., and Agell, N. (1994). Protein Kinase C Regulates Calmodulin Expression in NRK Cells Activated to Proliferate from Quiescence. Cell Calcium 16 (6), 446-454. doi:10.1016/0143-4160(94)90074-4

Burtness, B., Harrington, K. J., Greil, R., Soulières, D., Tahara, M., de Castro, G., Jr., et al. (2019). Pembrolizumab Alone or with Chemotherapy versus Cetuximab with Chemotherapy for Recurrent or Metastatic Squamous Cell Carcinoma of the Head and Neck (KEYNOTE-048): a Randomised, Open-Label, Phase 3 Study. Lancet 394 (10212), 1915-1928. doi:10.1016/s0140-6736(19)32591-7

Cahalan, M. D., and Chandy, K. G. (2009). The Functional Network of Ion Channels in T Lymphocytes. Immunol. Rev. 231 (1), 59-87. doi:10.1111/ j.1600-065X.2009.00816.x

Chapon, M., Randriamampita, C., Maubec, E., Badoual, C., Fouquet, S., Wang, S. F., et al. (2011). Progressive Upregulation of PD-1 in Primary and Metastatic Melanomas Associated with Blunted TCR Signaling in Infiltrating $\mathrm{T}$ Lymphocytes. J. Invest. Dermatol. 131 (6), 1300-1307. doi:10.1038/jid.2011.30 Chimote, A. A., Balajthy, A., Arnold, M. J., Newton, H. S., Hajdu, P., Qualtieri, J., et al. (2018). A Defect in KCa3.1 Channel Activity Limits the Ability of CD8+ $\mathrm{T}$ Cells from Cancer Patients to Infiltrate an Adenosine-Rich Microenvironment. Sci. Signal. 11 (527), eaaq1616. doi:10.1126/ scisignal.aaq1616

Chimote, A. A., Gawali, V. S., Newton, H. S., Wise-Draper, T. M., and Conforti, L. (2020). A Compartmentalized Reduction in Membrane-Proximal Calmodulin Reduces the Immune Surveillance Capabilities of CD8+ T Cells in Head and Neck Cancer. Front. Pharmacol. 11, 143. doi:10.3389/fphar.2020.00143

Chimote, A. A., Hajdu, P., Kottyan, L. C., Harley, J. B., Yun, Y., and Conforti, L. (2016). Nanovesicle-targeted Kv1.3 Knockdown in Memory T Cells Suppresses CD40L Expression and Memory Phenotype. J. Autoimmun. 69, 86-93. doi:10.1016/j.jaut.2016.03.004

Chimote, A. A., Hajdu, P., Kucher, V., Boiko, N., Kuras, Z., Szilagyi, O., et al. (2013). Selective Inhibition of KCa3.1 Channels Mediates Adenosine Regulation of the Motility of Human T Cells. J. Immunol. 191 (12), 6273-6280. doi:10.4049/jimmunol.1300702

Chimote, A. A., Hajdu, P., Sfyris, A. M., Gleich, B. N., Wise-Draper, T., Casper, K. A., et al. (2017). Kv1.3 Channels Mark Functionally Competent CD8+ TumorInfiltrating Lymphocytes in Head and Neck Cancer. Cancer Res. 77 (1), 53-61. doi:10.1158/0008-5472.CAN-16-2372

Chow, L. Q. M. (2020). Head and Neck Cancer. N. Engl. J. Med. 382 (1), 60-72. doi:10.1056/NEJMra1715715

Evrard, D., Hourseau, M., Couvelard, A., Paradis, V., Gauthier, H., Raymond, E., et al. (2020). PD-L1 Expression in the Microenvironment and the Response to Checkpoint Inhibitors in Head and Neck Squamous Cell Carcinoma. Oncoimmunology 9 (1), 1844403. doi:10.1080/2162402X.2020.1844403

Farhood, B., Najafi, M., and Mortezaee, K. (2019). CD8+ Cytotoxic T Lymphocytes in Cancer Immunotherapy: A Review. J. Cel. Physiol. 234 (6), 8509-8521. doi:10.1002/jcp.27782

\section{SUPPLEMENTARY MATERIAL}

The Supplementary Material for this article can be found online at: https://www.frontiersin.org/articles/10.3389/fphar.2021.742862/ full\#supplementary-material

Feske, S., Gwack, Y., Prakriya, M., Srikanth, S., Puppel, S. H., Tanasa, B., et al. (2006). A Mutation in Orail Causes Immune Deficiency by Abrogating CRAC Channel Function. Nature 441 (7090), 179-185. doi:10.1038/nature04702

Feske, S., Okamura, H., Hogan, P. G., and Rao, A. (2003). Ca2+/calcineurin Signalling in Cells of the Immune System. Biochem. Biophys. Res. Commun. 311 (4), 1117-1132. doi:10.1016/j.bbrc.2003.09.174

Feske, S., Skolnik, E. Y., and Prakriya, M. (2012). Ion Channels and Transporters in Lymphocyte Function and Immunity. Nat. Rev. Immunol. 12 (7), 532-547. doi: $10.1038 /$ nri3233

Feske, S., Wulff, H., and Skolnik, E. Y. (2015). Ion Channels in Innate and Adaptive Immunity. Annu. Rev. Immunol. 33, 291-353. doi:10.1146/annurev-immunol032414-112212

Kok, V. C. (2020). Current Understanding of the Mechanisms Underlying Immune Evasion from PD-1/pd-L1 Immune Checkpoint Blockade in Head and Neck Cancer. Front. Oncol. 10, 268. doi:10.3389/fonc.2020.00268

Newton, H. S., Gawali, V. S., Chimote, A. A., Lehn, M. A., Palackdharry, S. M., Hinrichs, B. H., et al. (2020). PD1 Blockade Enhances K+ Channel Activity, $\mathrm{Ca} 2+$ Signaling, and Migratory Ability in Cytotoxic T Lymphocytes of Patients with Head and Neck Cancer. J. Immunother. Cancer 8 (2), e000844. doi:10.1136/jitc-2020-000844

Ohya, S., and Kito, H. (2018). Ca2+-Activated K+ Channel KCa3.1 as a Therapeutic Target for Immune Disorders. Biol. Pharm. Bull. 41 (8), 1158-1163. doi:10.1248/bpb.b18-00078

Patsoukis, N., Li, L., Sari, D., Petkova, V., and Boussiotis, V. A. (2013). PD-1 Increases PTEN Phosphatase Activity while Decreasing PTEN Protein Stability by Inhibiting Casein Kinase 2. Mol. Cel. Biol. 33 (16), 3091-3098. doi:10.1128/ MCB.00319-13

Peng, W., Liu, C., Xu, C., Lou, Y., Chen, J., Yang, Y., et al. (2012). PD-1 Blockade Enhances T-Cell Migration to Tumors by Elevating IFN- $\gamma$ Inducible Chemokines. Cancer Res. 72 (20), 5209-5218. doi:10.1158/0008-5472.Can12-1187

Robbins, J. R., Lee, S. M., Filipovich, A. H., Szigligeti, P., Neumeier, L., Petrovic, M., et al. (2005). Hypoxia Modulates Early Events in T Cell Receptor-Mediated Activation in Human T Lymphocytes via Kv1.3 Channels. J. Physiol. 564 (Pt 1), 131-143. doi:10.1113/jphysiol.2004.081893

Seidel, J. A., Otsuka, A., and Kabashima, K. (2018). Anti-PD-1 and Anti-CTLA-4 Therapies in Cancer: Mechanisms of Action, Efficacy, and Limitations. Front. Oncol. 8, 86. doi:10.3389/fonc.2018.00086

Sforna, L., Megaro, A., Pessia, M., Franciolini, F., and Catacuzzeno, L. (2018). Structure, Gating and Basic Functions of the Ca2+-Activated K Channel of Intermediate Conductance. Curr. Neuropharmacol. 16 (5), 608-617. doi:10.2174/1570159x15666170830122402

Sharma, P., Hu-Lieskovan, S., Wargo, J. A., and Ribas, A. (2017). Primary, Adaptive, and Acquired Resistance to Cancer Immunotherapy. Cell 168 (4), 707-723. doi:10.1016/j.cell.2017.01.017

Srivastava, S., Choudhury, P., Li, Z., Liu, G., Nadkarni, V., Ko, K., et al. (2006a). Phosphatidylinositol 3-phosphate Indirectly Activates KCa3.1 via 14 Amino Acids in the Carboxy Terminus of KCa3.1. Mol. Biol. Cel. 17 (1), 146-154. doi:10.1091/mbc.e05-08-0763

Srivastava, S., Di, L., Zhdanova, O., Li, Z., Vardhana, S., Wan, Q., et al. (2009). The Class II Phosphatidylinositol 3 Kinase C2beta Is Required for the Activation of the K+ Channel KCa3.1 and CD4 T-Cells. Mol. Biol. Cel. 20 (17), 3783-3791. doi:10.1091/mbc.e09-05-0390

Srivastava, S., Li, Z., Ko, K., Choudhury, P., Albaqumi, M., Johnson, A. K., et al. (2006b). Histidine Phosphorylation of the Potassium Channel KCa3.1 by Nucleoside Diphosphate Kinase B Is Required for Activation of KCa3.1 and CD4 T Cells. Mol. Cel. 24 (5), 665-675. doi:10.1016/j.molcel.2006.11.012

Srivastava, S., Li, Z., Lin, L., Liu, G., Ko, K., Coetzee, W. A., et al. (2005). The Phosphatidylinositol 3-phosphate Phosphatase Myotubularin- Related Protein 6 
(MTMR6) Is a Negative Regulator of the Ca2+-Activated K+ Channel KCa3.1. Mol. Cel. Biol. 25 (9), 3630-3638. doi:10.1128/mcb.25.9.3630-3638.2005

Srivastava, S., Panda, S., Li, Z., Fuhs, S. R., Hunter, T., Thiele, D. J., et al. (2016). Histidine Phosphorylation Relieves Copper Inhibition in the Mammalian Potassium Channel KCa3.1. Elife 5, e16093. doi:10.7554/eLife.16093

Succaria, F., Kvistborg, P., Stein, J. E., Engle, E. L., McMiller, T. L., Rooper, L. M., et al. (2020). Characterization of the Tumor Immune Microenvironment in Human Papillomavirus-Positive and -negative Head and Neck Squamous Cell Carcinomas. Cancer Immunol. Immunother. 70, 1227-1237. doi:10.1007/ s00262-020-02747-w

Sullivan, M. R., Ugolini, G. S., Sarkar, S., Kang, W., Smith, E. C., McKenney, S., et al. (2020). Quantifying the Efficacy of Checkpoint Inhibitors on CD8+ Cytotoxic $\mathrm{T}$ Cells for Immunotherapeutic Applications via Single-Cell Interaction. Cell Death Dis. 11 (11), 979. doi:10.1038/s41419-020-03173-7

Sung, H., Ferlay, J., Siegel, R. L., Laversanne, M., Soerjomataram, I., Jemal, A., et al. (2021). Global Cancer Statistics 2020: GLOBOCAN Estimates of Incidence and Mortality Worldwide for 36 Cancers in 185 Countries. CA A. Cancer J. Clin. 71, 209-249. doi:10.3322/caac. 21660

Theodoraki, M. N., Yerneni, S. S., Hoffmann, T. K., Gooding, W. E., and Whiteside, T. L. (2018). Clinical Significance of PD-L1+ Exosomes in Plasma of Head and Neck Cancer Patients. Clin. Cancer Res. 24 (4), 896-905. doi:10.1158/10780432.Ccr-17-2664

Vaeth, M., Yang, J., Yamashita, M., Zee, I., Eckstein, M., Knosp, C., et al. (2017). ORAI2 Modulates Store-Operated Calcium Entry and T Cell-Mediated Immunity. Nat. Commun. 8, 14714. doi:10.1038/ncomms 14714

Wei, F., Zhong, S., Ma, Z., Kong, H., Medvec, A., Ahmed, R., et al. (2013). Strength of PD-1 Signaling Differentially Affects T-Cell Effector
Functions. Proc. Natl. Acad. Sci. U S A. 110 (27), E2480-E2489. doi:10.1073/pnas.1305394110

Zhou, J., Mahoney, K. M., Giobbie-Hurder, A., Zhao, F., Lee, S., Liao, X., et al. (2017). Soluble PD-L1 as a Biomarker in Malignant Melanoma Treated with Checkpoint Blockade. Cancer Immunol. Res. 5 (6), 480-492. doi:10.1158/23266066.Cir-16-0329

Zhou, K., Guo, S., Li, F., Sun, Q., and Liang, G. (2020). Exosomal PD-L1: New Insights into Tumor Immune Escape Mechanisms and Therapeutic Strategies. Front. Cel. Dev. Biol. 8, 569219. doi:10.3389/fcell.2020.569219

Conflict of Interest: The authors declare that the research was conducted in the absence of any commercial or financial relationships that could be construed as a potential conflict of interest.

Publisher's Note: All claims expressed in this article are solely those of the authors and do not necessarily represent those of their affiliated organizations, or those of the publisher, the editors and the reviewers. Any product that may be evaluated in this article, or claim that may be made by its manufacturer, is not guaranteed or endorsed by the publisher.

Copyright (C) 2021 Gawali, Chimote, Newton, Feria-Garzón, Chirra, Janssen, WiseDraper and Conforti. This is an open-access article distributed under the terms of the Creative Commons Attribution License (CC BY). The use, distribution or reproduction in other forums is permitted, provided the original author(s) and the copyright owner(s) are credited and that the original publication in this journal is cited, in accordance with accepted academic practice. No use, distribution or reproduction is permitted which does not comply with these terms. 